\title{
NNDC Chart of Nuclides
}

Alejandro Sonzogni

National Nuclear Data Center, Building 197D, Brookhaven National Laboratory, Upton, NY 11973-5000, USA

\begin{abstract}
The National Nuclear Data Center has recently developed an interactive chart of nuclides, http://www.nndc.bnl.gov/chart/, that provides nuclear structure and decay data. Since its implementation, it has proven to be one of the most popular web products. The information presented is derived from the ENSDF and Nuclear Wallet Card databases. Experimentally known nuclides are represented by a cell in chart with the number of neutrons on the horizontal axis and the number of protons on the vertical axis. The color of the cell is used to indicate the ground state half-life or the ground state predominant decay mode.
\end{abstract}

The Chart of Nuclides is a software product that allows searching and plotting nuclear structure and nuclear decay data interactively. The Chart of Nuclides provides an interface between web users and several databases containing nuclear structure, nuclear decay and some neutron-induced nuclear reaction information.

Using the Chart of Nuclides, it is possible to search for nuclear level properties (energy, half-life, spin-parity), gamma-ray information (energy, intensity, multipolarity, coincidences), radiation information following nuclear decay (energy, intensity, dose), and neutron-induced reaction data from the BNL-325 book (thermal cross section and resonance integral). The information provided by the Chart of Nuclides can be seen in tables, level schemes and an interactive chart of nuclides. The sections of the Chart of Nuclides related to levels, gammas and decay are obtained from the Evaluated Nuclear Structure Data File (ENSDF) [1] and the Nuclear Wallet Cards [2]. The Chart of Nuclides is periodically updated to reflect changes in ENSDF. The Chart of Nuclides is part of a number of applications developed by the NNDC [3]. A brief table showing some of the Chart of Nuclides contents can be found on the table 1:

Table 1. Some statistics on the Chart of Nuclides contents.

\begin{tabular}{ll}
\hline Number of nuclides & 3,166 \\
\hline Number of levels & 146,313 \\
\hline Number of levels with known T1/2 & 21,076 \\
\hline Number of levels with known $\mathrm{J}^{\pi}$ & 108,638 \\
\hline Number of gamma-rays & 216,368 \\
\hline Number of gamma-gamma coincidences & $2,622,671$ \\
\hline
\end{tabular}

From the ratio of the last two numbers, it is possible to deduce that on average, every single gamma ray is in cascade with 12 other gamma rays. This ratio will depend on each nuclide and will be smaller for nuclides in the vicinity of magic numbers.

Experimentally known nuclei are represented by a cell in a chart with the number of neutrons on the horizontal axis and the number of protons on the vertical axis. The color of the cell is used to indicate the ground state half-life or the ground state predominant decay mode. An example of the chart with the color of the cell according to half-life can be seen in figure 1 .

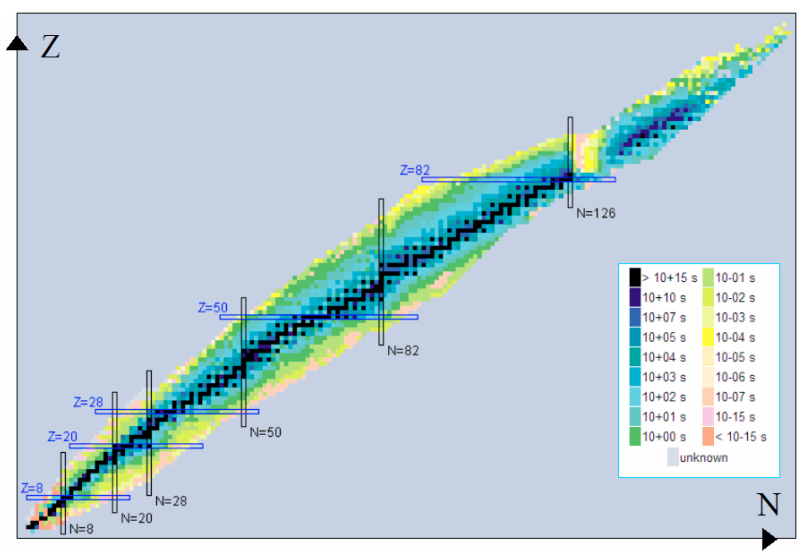

Fig. 1. Chart of Nuclei, the color of the cell is choosen according to half-life, the magic numbers 8, 20, 28, 50, 82 and 126 indicated.

The chart can be seen with 4 different zooming options. With the largest zoom value, the entire chart is displayed. For smaller zoom values, only a fraction of the chart is displayed; the nucleus at the center of the viewing window is marked with a magenta border; neutron (horizontal axis) and proton numbers (vertical axis) are indicated. When the zoom level is equal to 1, the name of the nucleus, the ground state halflife, the two decay modes with the highest probability and its natural abundance are displayed.

Ground and isomeric state information can be obtained by clicking on a given nucleus. Links to a list of levels, a level scheme, and decay radiation information are provided when available. Unless the zoom is at the largest value, clicking on a given nucleus also re-centers the chart. Further navigation possibilities can be obtained by using the arrows on the righttop corner of the window, as well as the "Nucleus" textbox under the zoom control. In order to optimize the transfer of information when using the interactive chart of nuclides, popup boxes (tool-tips) containing level information have been implemented. The tool-tips are triggered when the mouse is over a given nucleus on the chart. This effect is produced by using JavaScript programming with image mapping. The following picture shows this effect with an "onMouseOver" action. 


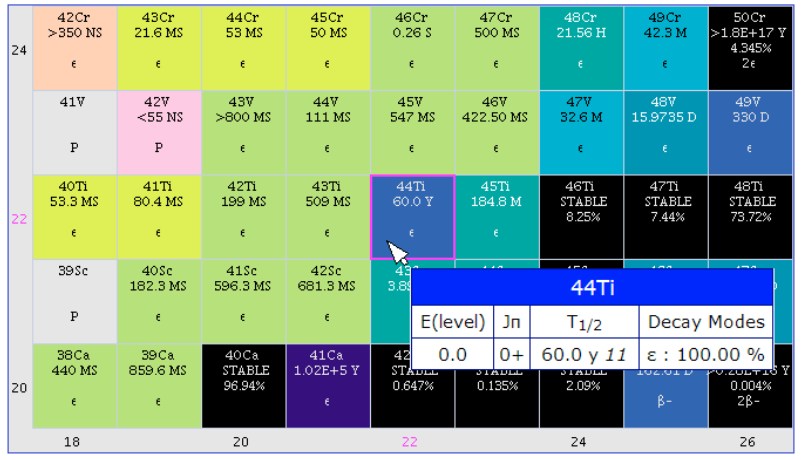

Fig. 2. Chart of nuclides centered on ${ }^{44} \mathrm{Ti}$ and colored according to half-life.

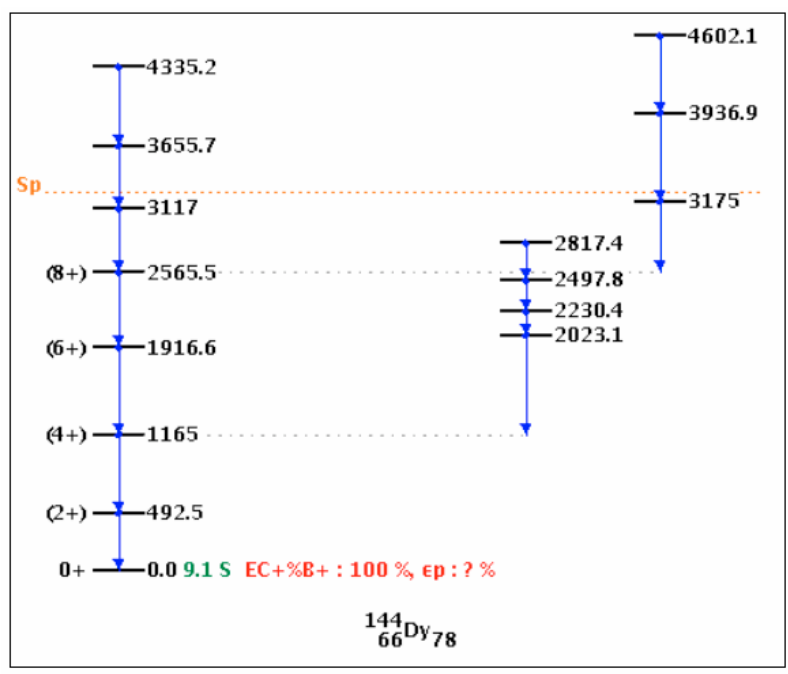

Fig. 3. Level Scheme for ${ }^{144} \mathrm{Dy}$, Sp indicates the proton separation energy, which is equal to $3265 \mathrm{keV}$, any level about this value is proton unbound.

As mentioned before, a link to a web page with a levels plot is provided after clicking on a given nucleus. An example of it can be seen in the figure 2 for ${ }^{144} \mathrm{Dy}$ : The level scheme is rendered in a single image. Most nuclei, however, have complex level schemes that won't fit in just one image. As a consequence, only selected parts of the level scheme should be plotted at a time using the interactive features provided. The neutron separation Energy (Sn) and the proton separation energy (Sp) are indicated in the level scheme. Decay schemes are produced in a similar way.

In the ENSDF file, uncertainties are given in the so-called "Nuclear Data Sheets" (NDS) style, where for instance if the gamma energy is $400.0+-0.22 \mathrm{keV}$, it is written in ENSDF as 400.022. Users can find this style quite difficult to follow, and hart to implement in numerical applications. The reason for the NDS style is that the ENSDF format was designed at a time where storage capabilities were extremely limited. Until recently, the Chart of Nuclides provided results in the Nuclear Data Sheets style only, but after extensive programming, the standard or regular way of quoting the uncertainties has been recently implemented.

In summary, a short description of the NNDC chart of Nuclides was presented. Because of its graphical interface, which facilitates the navigation, this application has become on the most popular web products at the NNDC. We do rely on users' feedback to add refinements to it; in fact, most of the defining features are the result of users request. As computer CPUs become faster and capable of handling multitasking more efficiently, new programming technologies that make the interplay between server and client task more effective have developed [3], which we look forward to implement.

This work was sponsored by the Office of Nuclear Physics, Office of Science, US Department of Energy, under contract DE-AC0298CH10886 with Brookhaven Science Associates, LLC.

\section{References}

1. J.K. Tuli, in Proceedings of the International Conference on Nuclear Data for Science and Technology, 2004, edited by R.C. Haight, M.B. Chadwick, T. Kawano, P. Talou, AIP Conference Proceedings 769 (2005), p. 265.

2. J.K. Tuli, 2005 edition of the Nuclear Wallet Cards, Brookhaven National Laboratory Report.

3. B. Pritychenko et al., Ann. Nucl. Energy 33, 390 (2006).

4. AJAX, Asynchronous JavaScript with XML, http://java.sun.com/blueprints/ajax.html. 